\title{
Electron diffraction studies of supersonic jets. II. Formation of benzene clusters
}

\author{
Richard K. Heenan, Edward J. Valente, and Lawrence S. Bartell \\ Department of Chemistry, University of Michigan, Ann Arbor, Michigan 48109 \\ (Received 9 September 1982; accepted 20 September 1982)

\begin{abstract}
Expansions of benzene at $1-10 \mathrm{~mol} \%$ in neon or helium with various nozzle types have produced clusters of benzene molecules. Preliminary comparisons with intensities from the bulk liquid and with model calculations based on solid state crystal structures suggest that on the average the clusters are probably considerably larger than a 13 molecule unit, and have a vibrational temperature on the order of $100-150 \mathrm{~K}$. Unlike clusters of spherical or quasispherical molecules previously found to pack in nearly crystalline arrays, benzene molecules appear to be unable to organize into regular arrays in the time of our experiments. Local order in clusters is similar but not identical to that in the solid, resembling that expected for a supercooled liquid. The present approach, exploiting several advantages of electron diffraction over alternative methods, shows promise as a new means of studying liquids or glasses.
\end{abstract}

\section{INTRODUCTION}

Many spectroscopic studies have been made of molecules cooled by seeding into supersonic expansions of a monatomic carrier gas. For the benzene molecule Duncan et al. ${ }^{1}$ detected by mass spectrometry clusters of up to 12 benzene monomers from $2 \mathrm{~mol} \%$ benzene in 5 atm helium. Further work by Hopkins et $a l .{ }^{2}$ and Langridge-Smith et al. ${ }^{3}$ revealed much about the electronic spectra of dimers, trimers, and tetramers, along with clues about their structures. We have attempted to obtain more direct structural information for larger benzene clusters by means of electron diffraction studies of benzene in helium or neon, at mole fractions of 0.01-0.1, with a variety of different nozzles. Prior research has shown that certain nozzle designs are much more effective at producing large clusters than are the nozzles most commonly used in spectroscopy. ${ }^{4,5}$ An attractive possibility, indeed, was the production of clusters large enough to be regarded as microdroplets of condensed matter, perhaps liquidlike because the short lifetimes before measurement might prove insufficient for the aggregates to crystallize. Electrons offer substantial advantages over $x$ rays and neutrons in the study of amorphous materials, provided the sample is thin enough for electrons to penetrate readily. Available for comparison with the present study are a number of $x$-ray and neutron studies of bulk benzene, liquid and solid, over a wide range of temperatures.

\section{EXPERIMENTAL}

We have recorded over 40 diffraction patterns of benzene in helium or neon as well as patterns for the individual pure gases. A representative selection of expansion conditions is listed in Table I. Due to the large number of experimental parameters to be varied only pairs of plates were taken under identical conditions in most cases. The first plate in each set of five placed in the camera was always exposed with no gas flow, in order to monitor extraneous scatter of the electron beam. Corrections for extraneous scattering, while made routinely, are almost insignificant. Monatomic carrier gases were passed over benzene in a brass tank which was either at room temperature or sus- pended in an ice bath. A glass wool plug in contact with the benzene served as a wick to increase surface area for saturation of the carrier, while helping to trap dust particles. Benzene was certified A.C.S. reagent grade from Fisher Scientific; helium $(99.995 \%)$ and neon (99.99\%) were obtained from Air Products Ltd.; all were used without further purification. Photographic plates were $4 \times 5$ in. Kodak medium projector slides with blackness corrections made as described elsewhere. $^{6}$ Intensities from plates taken under the same conditions were averaged. All intensities were then leveled by theoretical atomic scattering for benzene. Electron scattering factors were taken from Sellers et $a l .{ }^{7}$ Apparatus and data reduction procedures were described in detail in the previous paper of this series. ${ }^{8}$ Intensities for representative plates are avallable as supplemental material. ${ }^{9}$

\section{DIFFRACTION INTENSITIES}

One of the largest cluster contributions to experimental scattering that we have so far seen is shown in Fig. 1 for plate 358. The lowest trace of Fig. 1 represents the contribution to the intensity from intermolecular distances. It was obtained by subtraction of benzene monomer intensity (solid line in upper trace) from the total intensity (points in upper trace). Our least squares analysis program also subtracted a scaled, leveled intensity curve of the carrier gas, and applied a smooth background polynomial. ${ }^{8}$ Cluster diffraction patterns in Figs. 1 and 2 have data interpolated from our raw absorbances at intervals of $\Delta s=\pi / 20$, half of our normal interval for small molecules, consistent with the sharper features characteristic of clusters. Graph plotting programs join these points with local cubic polynomial functions.

In each case illustrated here the benzene monomer intensity was based on calculated amplitudes of vibration and shrinkages reported by Brooks et al. ${ }^{10}$ and a $\mathrm{C}-\mathrm{H}$ bond distance of $1.10 \AA$. Morse asymmetry parameters were taken to be $a=2.1 \AA^{-1}$ for C-C, 2.0 $\AA^{-1}$ for $\mathrm{C}-\mathrm{H}$ bonded distances, and zero for all others. The $\mathrm{C}-\mathrm{C}$ bond distance was permitted to vary, with a molecular model constrained to $D_{B n}$ symmetry, thereby allow- 
TABLE I. Experimental conditions for selected benzene in helium, benzene in neon, and pure benzene electron diffraction patterns.

\begin{tabular}{|c|c|c|c|c|c|c|c|c|}
\hline \multirow[b]{2}{*}{ Nozzle type } & \multicolumn{2}{|c|}{ Diam. (mm) } & \multirow[b]{2}{*}{$\begin{array}{l}\text { Length } \\
(\mathrm{mm})\end{array}$} & \multirow[b]{2}{*}{$\begin{array}{l}\text { Plate } \\
\text { no. }\end{array}$} & \multirow{2}{*}{$\begin{array}{c}\text { Relative } \\
\text { exposure } \\
(\mathbf{s})^{2}\end{array}$} & \multirow{2}{*}{$\begin{array}{c}\text { Total } \\
\text { pressure } \\
\text { (psi) }^{\mathbf{b}}\end{array}$} & \multirow{2}{*}{$\begin{array}{c}\mathrm{C}_{6} \mathrm{H}_{6} \\
\text { pressure } \\
\text { (psi) } \\
\end{array}$} & \multirow[b]{2}{*}{$\begin{array}{c}\text { Height } \\
\text { residual }\end{array}$} \\
\hline & Entrance & Exit & & & & & & \\
\hline Thin plate & 0.10 & $\infty$ & $\ldots$ & $87 / 8$ & 14 & $30 \mathrm{He}$ & 1.9 & 0.28 \\
\hline \multirow[t]{4}{*}{ Tubular } & 0.12 & 0.12 & 5.6 & $289 / 90$ & 19 & $30 \mathrm{He}$ & 0.5 & 0.13 \\
\hline & & & & $282 / 3$ & 14 & $30 \mathrm{He}$ & 1.9 & 0.25 \\
\hline & & & & $287 / 8$ & 7 & $70 \mathrm{He}$ & 0.5 & 0.34 \\
\hline & & & & $284 / 5$ & 8 & $70 \mathrm{He}$ & 1.9 & 0.67 \\
\hline \multirow{2}{*}{ Glass 4} & 0.141 & 2.6 & 33 & $375-378$ & 3 & $10 \mathrm{He}$ & 0.6 & 0.0 \\
\hline & & & & $382 / 3$ & 2 & $30 \mathrm{He}$ & 0.6 & 0.97 \\
\hline Glass 3 & 0.275 & 2.25 & 20 & $344 / 5$ & 0.5 & $13 \mathrm{He}$ & 1.9 & 0.54 \\
\hline \multirow{3}{*}{ Glass 1} & 0.145 & 2.15 & 20 & 360 & 0.8 & $20 \mathrm{Ne}$ & 2.0 & 0.66 \\
\hline & & & & 359 & 0.5 & $35 \mathrm{Ne}$ & 2.0 & 1.11 \\
\hline & & & & 358 & 0.4 & $50 \mathrm{Ne}$ & 2.0 & 1.38 \\
\hline \multirow[t]{2}{*}{ Glass 6} & 0.128 & 1.9 & 30 & $463 / 4$ & 1 & $30 \mathrm{Ne}$ & 1.6 & 0.63 \\
\hline & & & & $461 / 2$ & 0.6 & $70 \mathrm{Ne}$ & 1.6 & 0.98 \\
\hline Glass 1 & 0.145 & 2.15 & 20 & $302-305$ & 18 & $\mathrm{C}_{6} \mathrm{H}_{6}$ & 1.7 & 0.0 \\
\hline Bulk liquid & & & & $\mathrm{d}$ & & $\mathrm{C}_{6} \mathrm{H}_{6}$ & & 0.34 \\
\hline
\end{tabular}

${ }^{2}$ Relative exposure is the approximate exposure time for a peak absorbance of unity, for total scatter on the photographic plate, using our $R^{2}$ rotating sector and a $1 \mu \AA$ electron beam.

$1 \mathrm{~atm}=14.7 \mathrm{psi}=760 \mathrm{~mm} \mathrm{Hg}=101 \mathrm{kPa}$.

'This is the approximate height of the peak between $s=4.7$ and $5.8 \AA^{-1}$ on an $s M(s)$ plot of the trace representing intermolecular scattering or cluster intensity.

${ }^{d}$ Reference $14, x$-ray diffraction of room temperature liquid.

ing for small errors in the estimated effective camera height. Additional damping of the monomer intensity to take into account the broadened gas distribution was introduced as described previously. ${ }^{8}$ The limited range of scattering angle with our $R^{2}$ sector and often large underlying cluster intensity precluded a precise experimental determination of vibrational amplitudes for the monomer. To demonstrate the reliability of the calculated monomer intensity we show in Fig. 3 the fit to $s M(s)$ for pure benzene plates 302-305. These used a tapered glass nozzle with a broad gas jet at the electron beam (FWHM $\sim 4.5 \mathrm{~mm}$ ). The residual is comparable in magnitude to that which we might see in a con-

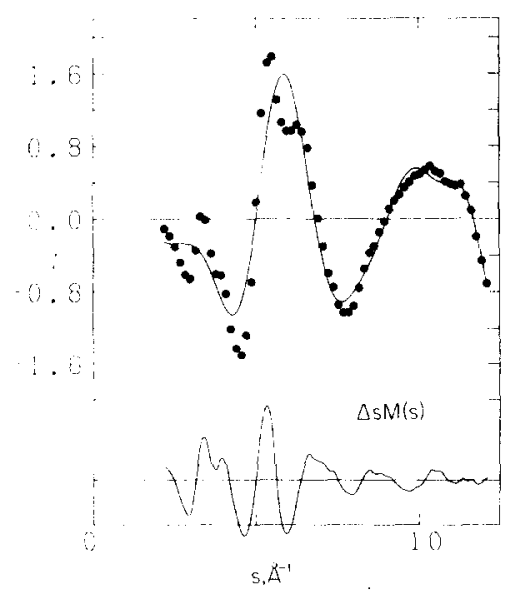

FIG. 1. Reduced intensity curves $s M(s)$. Points represent experimental data for plate 358 , benzene $(2.0$ psia) with neon at a reservoir pressure of $50 \mathrm{psia}$, using tapered glass nozzle 1 (see Table I). The lower trace is the experimental intensity less benzene monomer intensity (solid line in upper trace). ventional diffraction study, and is due more to systematic errors in scattering theory and experiment than to random noise.

\section{Optimization of cluster formation}

When clusters consist of microcrystals such as those observed, e.g., with argon, lead, and $\mathrm{SF}_{6},{ }^{11-14}$ it is

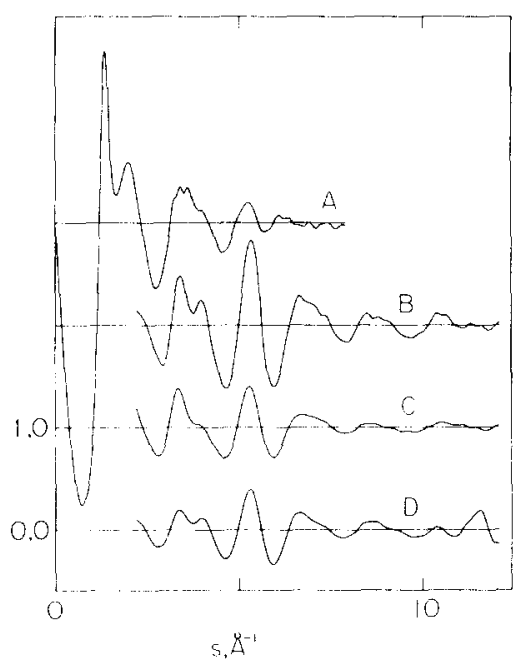

FIG. 2. (A) Experimental x-ray scattering $\boldsymbol{s H}_{d}(s)$ of Narten, Ref. 14 , for bulk liquid benzene at $298 \mathrm{~K}$. (B) - (D) are experimental intermolecular scattering $s M(s)$ from electron diffraction. Note that $H_{d}(s)$ and $M(s)$ treat inelastic scattering differently; however, the two functions are closely comparable; (B) Plate 358, also shown in Fig. 1. (C) Plate 360, benzene (2.0 psia) with neon at a reservoir pressure of 20 psia, using glass nozzle 1. (D) Plates $284 / 5$, benzene (1.9 psia) with helium at a reservoir pressure of 70 psia behind a tubular nozzle (see Table I). 


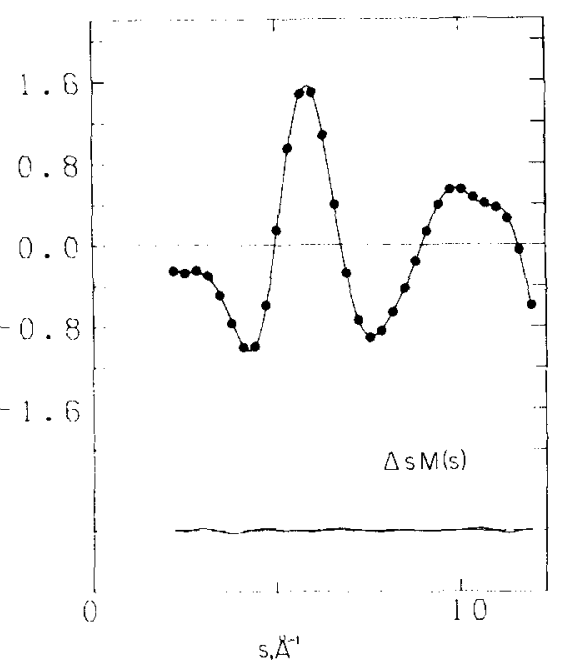

FIG. 3. Reduced intensity curves $s M(s)$ for pure benzene $(1.7$ psia) plates 302-305, using tapered glass nozzle 1 .

straightforward to estimate their diameters from the breadths of diffraction rings. Unfortunately, in the case of amorphous clusters such as benzene, no such simple gauge of cluster size is available from diffraction patterns. For present purposes we list in Table I the height of the prominent spike in $s M(s)$ between $s=4.7$ and $5.8 \AA^{-1}$, contributed by the clusters. For most of the plates exhibiting any sign of cluster intensity the pattern of cluster "residuals" is very similar in shape, though weaker, of course, when cluster formation is sparse. One feature displaying some variation in appearance is the double peak at $s \sim 3 \AA^{-1}$ as illustrated in Fig. 2 for plates 360,358 , and 284/5. The only difference between plates 360 and 358 was an increase in total pressure of neon from 20 psia, in the former case, to 50 psia. Experimental noise is becoming visible at high $s$ on the residual for 358 in which the benzene represents a smaller fraction of the total scattering. A spurious peak at $s=12 \AA^{-1}$ for plates $284 / 5$ arose due to problems with the background at the edge of the pattern. Inside the edge the curve is reliable and shows the largest cluster scattering we have seen with a tubular nozzle.

We record the helght of the feature at $s=5 \AA^{-1}$ as a measure of conversion of monomer to cluster only for lack of a better simple criterion. It should, however, be emphasized that this spike height cannot be taken as proportional to elther cluster size or fraction of monomer condensed. As will be shown later, the height depends as well on cluster structure and is a strong function of temperature. We shall also see that the cluster pattern is strongly correlated with that of liquid benzene as measured by $x$-ray diffraction. ${ }^{15}$ The fact that the greatest heights achieved for the $5 \AA^{-1}$ residual are about four times as intense as the corresponding feature for the bulk liquid is an Indication of our much lower temperature and not, of course, of higher conversion or larger size.

Several important features are indicated by the residual heights in Table I. Residual size for a particular nozzle increased with both total pressure and mole fraction of benzene. For each nozzle (except the thin plate) the highest pressure of carrier shown corresponds roughly to the maximum throughput that our pumps could take. In the case of neon the higher molecular weight of the carrier enabled us to reach greater pressures than with helium due to comparatively lower throughput, despite the greater capacity of the skimmer chamber diffusion pump for helium. Tapered glass nozzles seem more effective at producing clusters than a tubular nozzle of comparable throughput and diameter, though the difference may be small. Advantages of tapered nozzles are that they produce a better collimated gas jet and slow down expansions, thereby increasing the number of collisions during cooling. Some problems encountered due to condensation of benzene in the nozzle inlet were mentioned in a previous paper. ${ }^{8}$ Plates $87 / 8$, taken with a thin plate nozzle, seem similar to plates $282 / 3$, taken under comparable conditions with a tubular nozzle. Expansions of $n$-butane, for which we have more patterns available for comparison, also seem to show that in this range of conditions our tubular nozzle was no better than a thin plate nozzle at producing clusters. At first sight, tubular nozzles might have been expected to be somewhat more effective than thin plate nozzles because the effluent gas molecules from tubes are appreciably more collimated, under streamline flow conditions, and so might be expected to undergo more collisions during cooling. However, at the high pressures of the present work, a turbulent flow, choked by a sonic exit velocity, ${ }^{16}$ is reached and the output of a tubular nozzle approaches that of a thin plate aperture ${ }^{17}$

\section{INTERPRETATION OF DIFFRACTION PATTERNS}

Several questions may be posed about our benzene clusters such as their size and temperature, and whether their structure is best described as liquid, glass or crystalline. As a first attempt we have calculated in Fig. 4 intermolecular reduced intensities $s M(s)$ for some theoretical clusters using crystal lattice configurations. In crystalline benzene each molecule has 12 nearest neighbors, with molecular centers lying on a face-centered orthorhombic lattice. Molecular planes are twisted out of the planes defined by the crystal axes and the lattice group is primitive, space group Pbca. For illustrative calculations we chose two cluster sizes, the first with 13 molecules having a central benzene surrounded by its 12 nearest neighbors. The second, consisting of 55 molecules, was constructed from a 63 molecule block $2 a \times 2 b \times 2 c$, with all 8 vertices removed. Since nearest neighbor intermolecular distances dominate the scattering, one factor that determines the height of each cluster intensity is the average number of "closest contacts" per molecule. This increases from 2. 77 in $\left(\mathrm{C}_{6} \mathrm{H}_{6}\right)_{13}$ through 4.15 for $\left(\mathrm{C}_{6} \mathrm{H}_{6}\right)_{55}$ to 6.0 for an infinite lattice. Another major factor controlling the size of the cluster intensity is the chosen set of intermolecular amplitudes of vibration, which will be a function of temperature. To make comparisons with experimental patterns it is also necessary, in the absence of any other experimental information, to introduce some assumptions about the mole fraction of benzene mono- 


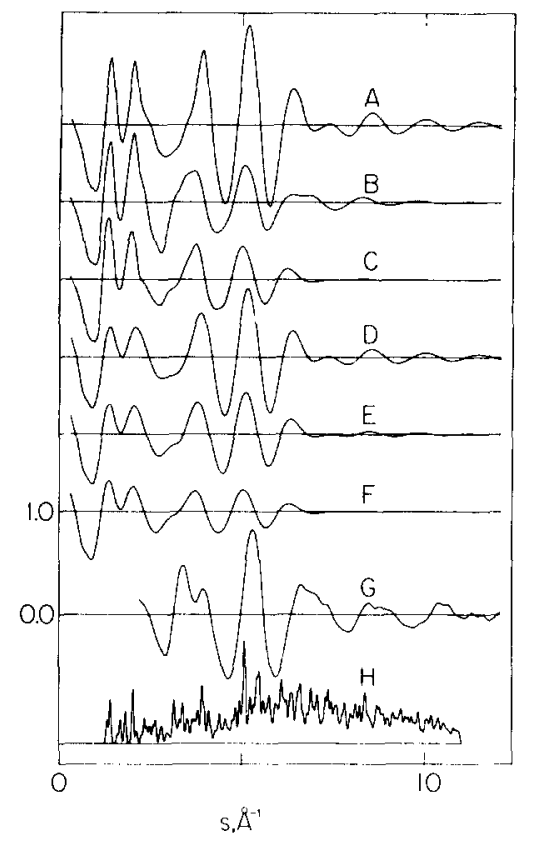

FIG. 4. Theoretical intermolecular scattering $s M(s)$ for benzene clusters based on crystal lattice parameters. A, B, and $C$ are for 55 molecules at 110,190 , and $270 \mathrm{~K}$, respectively. $D, E$, and $F$ are for 13 molecules, at 110,190 , and $270 \mathrm{~K}$. G. Experimental intermolecular scattering for plate 358 (also in Fig. 1). H. Simulation of powder diffraction pattern (arbitrary scale) for benzene crystals of size $\sim 200 \AA$, using crystal lattice parameters for $138 \mathrm{~K}, 1330$ reflections are allowed below $s=11 \AA^{-1}$. See the text for details.

mers remaining in the jet and to realize that there may be a distribution of cluster sizes.

A few comments are in order regarding the physical meaning of the parameter $l_{i j}$, the root-mean-square displacement we assign to the atom pair $i, j$. In amorphous cluster patterns, as in X-ray patterns of crystals, this so-called amplitude of vibration can correspond to a dynamic periodic vibrational displacement, to a disordered distribution of static or fluctuating internuclear distances between corresponding pairs, or to a combination of both. Several differences between clusters, which can be regarded as large, complex molecules, and crystals deserve to be mentioned. First, it is the rule for molecules that amplitudes $l_{i j}$ increase more or less regularly as the distance $r_{i j}$ between the atom pair increases. ${ }^{18}$ In crystal lattices on the other hand, the atoms are envisioned as oscillating about mean positions in a rather rigid lattice with the consequence that their internuclear amplitudes $l_{i}$, do not increase as $r_{i j}$ increases. To convert from the standard crystallographic mean-square amplitude $u_{i}^{2}$ of atom $i$ about its mean lattice site to the mean-square amplitude $l_{i j}^{2}$ required for characterization of amorphous materials, we proceeded as follows.

Our computational model was simplified by disregarding the directional properties of the crystallographic thermal ellipses. For spherically symmetric displacements the relation

$$
l_{i j}^{2}=u_{i}^{2}+u_{j}^{2}
$$

is expected to hold for atoms constrained in a truly periodic lattice. Thermal parameters $u_{i}^{2}$ and cell constants for crystalline benzene have been investigated at $270 \mathrm{~K}$ by Cox et al. by $\mathrm{x}$-ray diffraction, ${ }^{19}$ at 138 and $218 \mathrm{~K}$ by Bacon et al. using neutron diffraction, ${ }^{20}$ and between 272 and $276 \mathrm{~K}$ by Tohji $e$ t al. using energy dispersive x-ray diffraction. ${ }^{21}$ Typical root-mean-square intermolecular $\mathrm{C} \cdots \mathrm{C}$ amplitudes of vibration $l_{i}$, from these experiments were, respectively, $0.22,0.30$, and $0.37 \AA$ at 138,218 , and $270 \mathrm{~K}$. These agree fairly well at low temperatures with the inferences of Champeney and Woodhams from Mossbauer scattering. ${ }^{22}$ Their

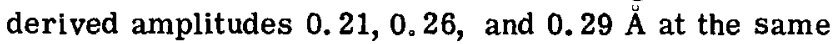
temperatures increase less rapidly. Curves in Fig. 4 correspond approximately to temperatures of 110,190 , and $270 \mathrm{~K}$. For 110 and $190 \mathrm{~K}$ we used atomic coordinates from the low temperature neutron studies but extrapolated the lattice constants to the slightly lower temperatures of our curves. Amplitudes of vibration were similarly treated, leading to principal $\mathrm{C} \cdot \mathrm{C}$ amplitudes of $0.20,0.275$, and $0.35 \check{A}$ for 110,190 , and $270 \mathrm{~K}$, respectively. If these amplitudes $l$ were applied to all $\mathrm{C} \cdot \mathrm{C}$ distances in the 55 molecule cluster, far more high frequency signals would be introduced into the cluster intensity than actually seen experimentally. Therefore all amplitudes were increased at longer distances to $l+0.1 \AA$ between 5.5 and $7.7 \AA$ and $l+0.2 \AA$ above $7.7 \AA$. To save computation only $C \cdots C$ and C ... H distances were included in the cluster calculation and similar distances were grouped at intervals of 0.05 to $10 \AA$, and $0.25 \AA$ intervals above that. In accordance with the neutron diffraction results the intermolecular C $\cdots$ H amplitude was set to $l+0.05 \AA$ for distances below $5.5 \AA$.

An alternative comparison between the cluster pattern and one expected for microcrystallites is provided by the synthesis of the crystal powder pattern. Calculations of crystal structure factors were made using the $138 \mathrm{~K}$ neutron diffraction lattice constants and molecular parameters. Electron scattering factors for crystals by Doyle and Cowley ${ }^{23}$ were leveled by our normal gas diffraction atomic scattering terms. Each powder ring was given a Gaussian profile corresponding to a crystal size of approximately $200 \AA$, comparable to that found for $\mathrm{SF}_{B}$ crystallites formed under roughly similar conditions. Ring contours were added together and then multiplied by $s$ to match the $s M(s)$ traces of Fig. 4 . The features of the simulation are in most cases superpositions of several powder rings, since there were in fact 1330 allowed reflections below $s=11 \breve{A}^{-1}$. As might be expected there is some correlation between the theoretical powder diffraction pattern and experimental cluster intensities, though not enough to indicate an appreciable concentration of well-ordered crystallites. Although we have not attempted in any of our calculations to include multiple scattering of electrons or the effects of absorption, we do not believe that such corrections would alter our qualitative conclusions.

Because the model cluster intensities of Fig. 4 depend predominantly upon nearest neighbor interactions, the height of the $s=5 \AA^{-1}$ spike scales closely with the num- 
ber of closest contacts per molecule if the thermal parameters are fixed. An examination of the curve for plate 358 in Figs. 1 and 2, Indicates that the rate of damping of the signal with $s$ agrees best with the lowest temperature models. Unless the temperature were lowered to below $80 \mathrm{~K}$, the height of the curve gives a lower bound on the average cluster size as 13 molecules and probably much larger. If a significant fraction of benzene remained uncondensed the clusters must certainly have contained still more molecules. As for the other curves in Fig. 2, conclusions are harder to reach, though it seems that plates $284 / 5$ are similar to 358 , but with more benzene monomer, whereas 360 may have a higher temperature. It is significant that none of the models proposed so far have produced the double peak around $s=3 \AA^{-1}$. However, both this feature and the $5 \AA^{-1}$ spike bear a resemblance to the corresponding features of the intermolecular pattern for liquid benzene derived by $x$-ray diffraction. ${ }^{15}$ The principal difference between the $\mathrm{x}$-ray $s M(s)$ curve and that for electrons is that the $x$-ray curve, taken at room temperature, damps considerably more rapidly with $s$, as is evident in Fig. 2. It also appears that, despite the complication of scattering by the carrier gas, electron diffraction patterns have less noise arising from counting statistics. Moreover, because of the low temperatures achieved in the supersonic expansion, the electron diffraction patterns contain conspicuously more structural information to help characterize the amorphous material produced.

\section{CONCLUSIONS}

It is premature to draw firm conclusions about the nature of the benzene clusters produced by supersonic expansions with a carrier gas. A provisional interpretation of results may be justified at this point, in part, to stimulate further research into what appears to be a fruitful method. It is clear that the clusters have a short-range order resembling that found in crystals. The actual diffraction patterns, however, are definitely not those of microcrystallites of the sort observed, e.g., in simllar experiments with $\mathbf{S F}_{\mathbf{6}}$. Instead the patterns appear to be a much more slowly damped modification of the pattern of the bulk liquid recorded at room temperature. The decreased damping and hence, greatly increased signal, is indicative of curtailed molecular motion at a low temperature. We speculate that our clusters may perhaps be regarded as strongly supercooled liquid microdrops, or glasses, too cold to overcome the activation barrier to crystallization in the few dozen microseconds of their lifetime before they are probed by the electron beam. Atoms, such as argon $^{11}$ and lead, ${ }^{12}$ and quasispherical molecules such as $\mathrm{SF}_{6,}{ }^{13,14}$ have been found to organize into more or less crystalline clusters in supersonic expansions. It is likely that molecules of lower symmetry, such as benzene, crystallize less readily when rapidly condensed. We have observed a similar reluctance of $n$-alkanes to crystallize. In view of the fact that liquids and glasses constitute the most poorly understood commonly occuring phases of matter, ${ }^{24}$ our approach to research in this area, as outlined in the foregoing, seems to hold great promise. Electron diffraction, where applicable, has many advantages over $x$-ray and neutron diffraction in resolving power, counting statistics, and sensitivity. To date is has been hampered in the study of liquids and glasses by the short range of electrons in matter. Microdrops in supersonic jets circumvent this trouble.

Further information, both experimental and theoretical, is needed before the nature of the microdrops is securely established. A rotating sector has been built to record diffraction patterns to lower scattering angles. In addition, a statistical mechanics study is being carried out on supercooled benzene as a function of density, temperature, and intermolecular potential. Calculated radial distribution functions transformed to electron diffraction intensities should greatly facilitate interpretation of our patterns. Calculations are based on the reference interaction site model (RISM), introduced by Lowden and Chandler, ${ }^{25}$ in the variant described by Johnson and Hazoume. ${ }^{26}$ We have recently become aware of some molecular mechanics calculations by Williams, ${ }^{27}$ on small benzene clusters, from which we shall compute intensity functions for a future publication. A successful resolution of the experimental findings would elucidate not only the structure and dynamics of cluster formation in supersonic jets but also the intermolecular potentials governing packing in clusters.

\section{ACKNOWLEDGMENTS}

This research was supported by grants from the National Science Foundation. We thank Martin Shetter, Anding Jin, and Thomas Trull for their assistance with the recording and processing of electron diffraction data. We are indebted to A. H. Narten and E. Johnson for helpful discussions and the use of their RISM program. A generous allocation of computer resources from the University of Michigan Computer Center is gratefully acknowledged.

${ }^{1}$ M. A. Duncan, T. G. Dietz, M. G. Liverman, and R. E. Smalley, J. Phys. Chem. 85, 7 (1981).

${ }^{2}$ J. B. Hopkins, D. E. Powers, and R. E. Smalley, J. Phys. Chem. 85, 3739 (1981).

${ }^{3}$ P. R. R. Langridge-Smith, D. V. Brumbaugh, C. A. Haynam, and D. H. Levy, J. Phys. Chem. 85, 3742 (1981).

${ }^{4} \mathrm{O}$. F. Hagena and $\mathrm{H}$. von Wedel, Rarefied Gas Dynamics, 9th Symp., edited by M. Becker and M. Fiebig (DFVLR, PorzWahn, Germany, 1974); O. F. Hagena and W. Obert, J. Chem. Phys. 56, 1793 (1972).

${ }^{5}$ O. Abraham, S. S. Kim, and G. D. Stein, J. Chem. Phys. 75,402 (1981).

${ }^{6}$ S. R. Goates, Thesis, University of Michigan, 1981; S. R. Goates and L. S. Bartell, J. Chem. Phys. 77, 1866, 1874 (1982).

${ }^{7}$ H. L. Sellers, L. Schafer, and R. A. Bonham, J. Mol. Struct. 49, 125 (1978).

${ }^{8}$ L. S. Bartell, R. K. Heenan, and M. Nagashima, J. Chem. Phys. (submitted).

${ }^{9}$ See AIP document no. PAPS JCPSA-78-243-5 for 5 pages of tables containing experimental intensities, background functions, and intermolecular scattering for plates discussed in this work. Order by PAPS number and journal reference from American Institute of Physics, Physics Auxiliary Publication Service, 335 East 45th Street, New York, N. Y. 10017. 
The price is $\$ 1.50$ for a microfiche, or $\$ 5$ for a photocopy. Airmail is additional. Make checks payable to the American Institute of Physics.

${ }^{10}$ W. V. F. Brooks, S. J. Cyvin, and P. C. Kvande, J. Phys. Chem. 69, 1489 (1965).

${ }^{11} \mathrm{~J}$. Farges, M. F. deFeraudy, B. Raoult, and G. Torchet, J. Phys. Paris, 41, C3-263 (1980).

${ }^{12}$ A. Yokozeki, J. Chem. Phys. 68, 3766 (1978).

${ }^{13}$ B. G. DeBoer, S. S. Kim, and G. D. Stein, Rarefied Gas Dynamics, 11th Symp., edited by R. Campargue (Commissiariat a l'Energie Atomique, Paris, 1979).

${ }^{1}$ E. J. Valente, R. K. Heenan, and L. S. Bartell (unpublished research).

${ }^{15}$ A. H. Narten, J. Chem. Phys. 67, 2102 (1977).

${ }^{16} \mathrm{~L}$. Prandt1, Essentials of Fluid Dynamics, (Blackie, London, 1952); A. D. Young and N. E. Winterbottom, Report No. 2068 (Royal Aircraft Establishment, England, Nov. 1942).

${ }^{17} \mathrm{E}$. J. Valente and L. S. Bartell (unpublished research).

${ }^{18}$ S. J. Cyvin and B. N. Cyvin, Spectrose. Lett. 14, 287
(1981); S. J. Cyvin, J. Brunvoll, B. N. Cyvin, and V. S. Mastryukov, Z. Naturforsch. Teil A 34, 1512 (1979).

${ }^{19}$ E. G. Cax, D. W. J. Cruickshank, and J. A. S. Smith, Proc. R. Soc. London Ser. A 247, 1 (1958).

${ }^{20}$ G. E. Bacon, N. A. Curry, and S. A. Wilson, Proc. R. Soc. London, Ser. A 279, 98 (1964).

${ }^{21} \mathrm{~K}$. Tohji, N. Nishikawa, and Y. Murata, J. Appl. Phys. Jpn. 19, L365 (1980).

${ }^{22}$ D. C. Champeney and F. W. D. Woodhams, J. Phys. B 620 (1968).

${ }^{23} \mathrm{P}$. A. Doyle and J. M. Cowley, in International Tables for $X$-Ray Crystallography (Kynoch, Birmingham, England, 1974), Vol. IV.

${ }^{24}$ G. D. Stein, Phys. Teach. 17, 503 (1979).

${ }^{25}$ L. J. Lowden and D. Chandler, J. Chem. Phys. 61, 5228 (1974).

${ }^{26}$ E. Johnson and R. P. Hazoume, J. Chem. Phys. 70, 1599 (1979).

${ }^{27}$ D. E. Williams, Acta Crystallogr. Sect. A 36, 715 (1980). 